\title{
Effects of Variable Viscosity and Thermal Conductivity on Unsteady Free Convective Heat and Mass Transfer MHD Flow of Micropolar Fluid with Constant Heat Flux through a Porous Medium
}

\author{
Parash Moni Thakur \\ Department of Mathematics \\ D. K. D. College, Deragaon \\ Assam, India
}

\author{
G. C. Hazarika \\ Dibrugarh University \\ Dibrugarh \\ Assam, India
}

\begin{abstract}
The effects of temperature dependent viscosity and thermal conductivity on magneto-hydrodynamic unsteady free convective heat and mass transfer flow of an incompressible micropolar fluid through a porous medium along an infinite vertical plate is studied. A magnetic field of uniform strength is assumed to be applied which makes an angle $\alpha$ with the plane transverse to the direction of the main flow. A similarity parameter $\sigma$ has been introduced and the suction velocity is considered to be inversely proportional to this time dependent parameter. The partial differential equations governing the flow, heat and mass transfer of the problem are transformed into dimensionless form of ordinary differential equations by using similarity substitutions. The governing boundary value problems are then solved numerically using Runge-Kutta shooting method. The effects of various parameters, viz. viscosity parameter, thermal conductivity parameter, mass transfer parameter, coupling constant parameter, Grashoff number, Prandtl number, Schmidt number and magnetic parameter on velocity, secondary velocity, micro-rotation, temperature and concentration field are obtained and presented graphically. The Skin-friction, Nusselt number and Sherwood number are also computed and presented in table.
\end{abstract}

\section{Keywords}

Micropolar fluid, variable viscosity, thermal conductivity, heat and mass transfer, MHD Flow, Hall Current

\section{INTRODUCTION}

The micropolar fluid, a subclass of micro-fluid, developed by Eringen [1], derives from the need to model the flow of fluids that contain rotating micro-constituents. The micro polar fluids can support couple stress and body couple only in which each particle has a finite size and constitutes a micro structure that can rotate about the centre of the volume element described by the micro-rotation vector. If a conductor or a semi conductor has current flowing in it because of an applied electric field and a transverse magnetic field is applied, there develops a component of electric field in the direction orthogonal to both the applied electric field and magnetic field, resulting in a voltage difference between the sides of the conductor. Due to the gyration and drift of charged particles, the conductivity parallel to the electric field is reduced and the current is induced in the direction normal to both electric and magnetic fields. This phenomenon is known as the Hall Effect. When the strength of applied magnetic field is very strong, one cannot neglect the effect of Hall currents. This effect can be taken into account within the range of magneto-hydro dynamical approximation. Several investigations have made theoretical and experimental studies of micropolar flow in the presence of a transverse magnetic field during the last decades. Gorla et al. [2] investigated the magneto hydrodynamic free convection boundary layer flow of a thermo micropolar fluid over a vertical plate. The effect of hall currents on the magneto hydrodynamic boundary layer flow past a semi-infinite flat plate was studied by Katagiri [4]. Hossain [5] investigated the effect of hall current on unsteady hydro magnetic free-convection flow near an infinite vertical porous plate. Sattar and Hossain [6] studied the unsteady hydromegnetic free convection flow with hall current and mass transfer along an accelerated porous plate with time dependent temperature and concentration. Sarma and Hazarika [8] studied effects of variable viscosity and thermal conductivity on combined free-forced convection and mass transfer flow passed a vertical porous plate. Following the works of Sattar et al. [7] Sarma and Hazarika [9] investigated the effects of variable viscosity and thermal conductivity on free convective heat and mass transfer flow with constant heat flux through a porous medium.

The main objective of our present work is to extend the work of Sarma and Hazarika [9] for the study of effects of variable viscosity and thermal conductivity on unsteady free convective heat and mass transfer MHD flow of micropolar fluid with constant heat flux through a porous medium. Viscosity and thermal conductivity are assumed to be inverse linear functions of temperature. The governing partial differential equations are reduced in to ordinary differential equations by similarity transformations. The problem is then solved numerically using Runge-kutta shooting algorithm with iteration process.

\section{MATHEMATICAL FORMULATION OF THE PROBLEM:}

We consider an unsteady free convective flow of an incompressible micropolar electrically conducting fluid through a porous medium along a semi-infinite porous vertical plate in presence of magnetic field. The plate is at constant temperature $T_{w}$. The temperature of the fluid far from the plate is $T_{\infty}$. The $x$-axis is taken along the plate in upward direction and the $y$-axis is taken normal to it. The plate is parallel to the direction which is also the direction of gravity but directed vertically upward. $N$ is the component of micro-rotation perpendicular to the $x y$-plane. The level of species concentration is assumed to be very low and hence species thermal diffusion and diffusion thermal energy effects can be neglected. A strong magnetic field of intensity $B$ is imposed in a direction that makes an angle $\alpha$ with the plane transverse to the vertical plate which is 
assumed to be non-conducting, such that $B=$ $\left(0, \delta_{1} B_{0}, \sqrt{\left(1-\delta_{1}^{2}\right)} B_{0}\right)$ where $\delta_{1}=\cos \alpha$, $\mathrm{B}_{0}=$ applied magnetic field. If $\delta_{1}=1$ the imposed magnetic field is parallel to the $y$-direction and if $\delta_{1}=0$ the magnetic field is parallel to the plate. The magnetic Reynolds number of the flow is taken to be small enough so that the induced magnetic field is negligible compared to the applied magnetic field and the magnetic lines of force are fixed relative to the fluid, Shercliff [11]. The plate is assumed to be non-conducting hence $\mathrm{J}_{y}=0$ at the plate and hence zero everywhere. Neglecting electron pressure and ion slip we have from Ohm's law

$\mathrm{J}_{x}=\frac{p \delta_{1} \mu_{c} B_{0}}{\rho\left(1+m^{2} \delta_{1}^{2}\right)}\left(m \delta_{1} u-w\right), \mathrm{J}_{y}=$ $\frac{p \delta_{1} \mu_{c} B_{0}}{\rho\left(1+m^{2} \delta_{1}{ }^{2}\right)}\left(u+m \delta_{1} w\right)$, where $m=\omega_{e} \tau_{e}$ is the hall parameter.

\subsection{Basic Equations: Equation of continuity:}

$$
\frac{\partial v}{\partial y}=0
$$

\section{Momentum equations:}

$$
\begin{aligned}
& \frac{\partial u}{\partial t}+v \frac{\partial u}{\partial y}=g_{0} \beta\left(T-T_{\infty}\right)+g_{0} \beta^{*}\left(C-C_{\infty}\right) \\
& +\frac{\partial}{\partial y}\left(v \frac{\partial u}{\partial y}\right)+\frac{\kappa}{\rho}\left(\frac{\partial N}{\partial y}+\frac{\partial^{2} u}{\partial y^{2}}\right)-\frac{v u}{K^{\prime}} \\
& -\frac{p \delta_{1} \mu_{c}{ }^{2} B_{0}{ }^{2}}{\rho\left(1+m^{2} \delta_{1}{ }^{2}\right)}\left(u+m \delta_{1} w\right) \\
& \frac{\partial w}{\partial t}+v \frac{\partial w}{\partial y}=\frac{\partial}{\partial y}\left(v \frac{\partial w}{\partial y}\right)+\frac{\kappa}{\rho}\left(\frac{\partial N}{\partial y}+\frac{\partial^{2} w}{\partial y^{2}}\right) \\
& +\frac{p \delta_{1} \mu_{c}{ }^{2} B_{0}{ }^{2}}{\rho\left(1+m^{2} \delta_{1}{ }^{2}\right)}\left(m \delta_{1} u-w\right)
\end{aligned}
$$

\section{Angular momentum equation:}

$$
\frac{\partial N}{\partial t}+v \frac{\partial N}{\partial y}=\frac{\gamma}{\rho \mathrm{j}} \frac{\partial^{2} N}{\partial y^{2}}-\frac{\kappa}{\rho \mathrm{j}}\left(2 N+\frac{\partial u}{\partial y}\right)
$$

\section{Energy equation:}

$$
\begin{array}{r}
\frac{\partial T}{\partial t}+v \frac{\partial T}{\partial y}=\frac{1}{\rho c_{\mathrm{p}}} \frac{\partial}{\partial y}\left(\lambda \frac{\partial T}{\partial y}\right)+\frac{\kappa+\mu}{\rho c_{\mathrm{p}}}\left[\left(\frac{\partial u}{\partial y}\right)^{2}+\right. \\
\left.\left(\frac{\partial w}{\partial y}\right)^{2}\right]
\end{array}
$$

\section{Equation of mass transfer:}

$$
\frac{\partial C}{\partial t}+v \frac{\partial C}{\partial y}=\frac{1}{\mathrm{~S}_{\mathrm{c}}} \frac{\partial}{\partial y}\left(v \frac{\partial C}{\partial y}\right)
$$

Following Gorla [2] we have supposed that $\gamma=$ $\left(\mu_{\infty}+\frac{\kappa}{2}\right) j=\mu_{\infty}\left(1+\frac{K_{1}}{2}\right) j, \quad$ where $K_{1}=\frac{\kappa}{v_{\infty} \rho}$, coupling constant parameter.

$$
\left.\begin{array}{lll}
y=0: & u=w=0, N=0, \frac{\partial T}{\partial y}=-\frac{q}{\lambda_{\infty}}, \quad C=C_{w} \\
y \rightarrow \infty: & u \rightarrow 0, w \rightarrow 0, N \rightarrow 0, T \rightarrow T & C \rightarrow C \infty
\end{array}\right\} \text { (7) }
$$

A similarity parameter $\sigma=\sigma(\mathrm{t})$ is introduced in order to make equations (1) - (6) similar where $\sigma$ is in fact a time dependent length scale so that the governing equations could be transformed into a similar form in time. Using this length scale the solution of the equation (1) is considered to be

$$
v=-v_{0} \frac{v_{\infty}}{\sigma}
$$

where $v=$ velocity vector , $v_{0}=$ suction parameter and $v_{0}>0$.The negative sign indicates that the suction is towards the plate. It is to be mentioned that the basic flow in the medium is entirely due to buoyancy force caused by temperature difference between the wall and the medium.

We introduce the following non-dimensional quantities:

$$
\left.\begin{array}{c}
\eta=\frac{y}{\sigma}, f(\eta)=\frac{u}{U_{0}}, \varphi(\eta)=\frac{w}{U_{0}}, \theta(\eta)=\frac{\lambda_{\infty}\left(T-T_{\infty}\right)}{\mathrm{q} \sigma}, \\
g(\eta)=\frac{C-C_{\infty}}{C_{w}-C_{\infty}}, h(\eta)=\frac{\sigma \mathrm{N}}{U_{0}}
\end{array}\right\} \ldots
$$

Following Lai and Kulacki [3] we assume that the viscosity and thermal conductivity are inverse linear functions of temperature, i.e.

$$
\left.\begin{array}{rl}
\frac{1}{\mu}= & \frac{1}{\mu_{\infty}}\left[1+\delta\left(T-T_{\infty}\right)\right], \text { or } \frac{1}{\mu}=a\left(T-T_{c}\right) \\
& \text { where } a=\frac{\delta}{\mu_{\infty}} \text { and } T_{c}=T_{\infty}-\frac{1}{\delta} \\
\frac{1}{\lambda}= & \frac{1}{\lambda_{\infty}}\left[1+\xi\left(T-T_{\infty}\right)\right], \text { or } \frac{1}{\lambda}=b\left(T-T_{r}\right) \\
& \text { where } b=\frac{\xi}{\lambda_{\infty}} \text { and } T_{r}=T_{\infty}-\frac{1}{\xi}
\end{array}\right\}
$$

where $a, b, T_{r}$ and $T_{c}$ are constants and their values depend on the reference state and thermal properties of the fluid i.e. $v$ and $\lambda$.

$$
\begin{aligned}
& \text { Then we get } \quad v=-v_{\infty} \frac{\theta_{c}}{\theta-\theta_{c}}, \quad \lambda=-\lambda_{\infty} \frac{\theta_{r}}{\theta-\theta_{r}}, \\
& \left.\theta_{c}=\frac{T_{c}-T_{\infty}}{T_{w}-T_{\infty}}, \theta_{r}=\frac{T_{r}-T_{\infty}}{T_{w}-T_{\infty}} \quad\right\}
\end{aligned}
$$

Introducing the above transformations in equations (2) - (6), we have the following non dimensional equations $\left(1+K_{1} \frac{\theta_{c}-\theta}{\theta_{c}}\right) f^{\prime \prime}=\left[G_{r} \theta+G_{c} g+\left(\frac{\eta \sigma}{v_{\infty}} \frac{d \sigma}{d t}+v_{0}\right) f^{\prime}+K_{1} h^{\prime}-\right.$ $\left.M \delta_{1}\left(\frac{f}{1+m^{2} \delta_{1}{ }^{2}}+\frac{m \delta_{1} \varphi}{1+m^{2} \delta_{1}{ }^{2}}\right)\right] \frac{\theta-\theta_{c}}{\theta_{c}}-K f+\frac{\theta^{\prime} f^{\prime}}{\theta-\theta_{c}}$

$$
\begin{aligned}
& \left(1+K_{1} \frac{\theta_{c}-\theta}{\theta_{c}}\right) \varphi^{\prime \prime}=\left[\left(\frac{\eta \sigma}{v_{\infty}} \frac{d \sigma}{d t}+v_{0}\right) \varphi^{\prime}+K_{1} h^{\prime}+\right. \\
& \left.M \delta_{1}\left(\frac{m \delta_{1} f}{1+m^{2} \delta_{1}{ }^{2}}-\frac{\varphi}{1+m^{2} \delta_{1}{ }^{2}}\right)\right] \frac{\theta-\theta_{c}}{\theta_{c}}+\frac{\theta^{\prime} \varphi^{\prime}}{\theta-\theta_{c}}
\end{aligned}
$$

$$
\left(2+K_{1}\right) h^{\prime \prime}=K_{2}\left(4 h+2 f^{\prime}\right)-2\left(\frac{\eta \sigma}{v_{\infty}} \frac{d \sigma}{d t}+v_{0}\right) h^{\prime}
$$

$$
\theta^{\prime \prime}=P_{r}\left[\left(\eta \theta^{\prime}-\theta\right) \frac{\sigma}{v_{\infty}} \frac{d \sigma}{d t}+v_{0} \theta^{\prime}\right] \frac{\theta-\theta_{r}}{\theta_{r}}+\frac{\theta^{\prime 2}}{\theta-\theta_{r}}
$$$$
+P_{r} E_{c} \frac{\theta-\theta_{r}}{\theta_{r}}\left(\frac{\theta_{c}}{\theta_{c}-\theta}+K_{1}\right)
$$

$g^{\prime \prime}=S_{c}\left[\left(\frac{\eta \sigma}{v_{\infty}} \frac{d \sigma}{d t}+v_{0}\right) g^{\prime}\right] \frac{\theta-\theta_{c}}{\theta_{c}}+\frac{g^{\prime} \theta^{\prime}}{\theta-\theta_{c}}$

The dimensionless numbers are as follows: 


$$
\left.\begin{array}{c}
K_{1}=\frac{\kappa}{\rho v_{\infty}}, G_{r}=\frac{g_{0} \beta q \sigma^{3}}{v_{\infty} \lambda_{\infty} U_{0}}, G_{c}=\frac{g_{0} \beta^{*}\left(C_{w}-C_{\infty}\right) \sigma^{2}}{v_{\infty} U_{0}}, \\
M=\frac{p \mu_{c}^{2} \sigma^{2} B_{0}{ }^{2}}{\rho v_{\infty}}, K=\frac{\sigma^{2}}{K^{\prime}} \\
S_{c}=\frac{v_{\infty}}{D}, P_{r}=\frac{\rho c_{p} v_{\infty}}{\lambda_{\infty}}, \quad E_{c}=\frac{\lambda_{\infty} U_{0}^{2}}{\sigma q c_{p}} \quad K_{2}=\frac{\sigma^{2} \kappa}{j \rho v_{\infty}}
\end{array}\right\}
$$

Following the works of Sattar and Hussain [6] the

similarity condition requires that

$$
\frac{\sigma}{v_{\infty}} \frac{d \sigma}{d t}=2
$$

Integrating (18) we get, $\sigma=2 \sqrt{\nu_{\infty} t}$, which corresponds with the usual scaling factor for unsteady boundary layer flows, Schlichiting [10]. Using (18) in (12) - (16) and writing $2 \zeta=2 \eta+v_{0}$, finally we get

$$
\begin{aligned}
\left(1+K_{1} \frac{\theta_{c}-\theta}{\theta_{c}}\right) f^{\prime \prime} & =\left[\begin{array}{l}
G_{r} \theta+G_{c} g+2 \zeta f^{\prime}+K_{1} h^{\prime} \\
-M \delta_{1}\left(\frac{f}{1+m^{2} \delta_{1}{ }^{2}}+\frac{m \delta_{1} \varphi}{1+m^{2} \delta_{1}{ }^{2}}\right)
\end{array}\right] \frac{\theta-\theta_{c}}{\theta_{c}} \\
& -K f+\frac{\theta^{\prime} f^{\prime}}{\theta-\theta_{c}}
\end{aligned}
$$

$$
\begin{aligned}
& \begin{array}{l}
\left(1+K_{1} \frac{\theta_{c}-\theta}{\theta_{c}}\right) \varphi^{\prime \prime}=\left[2 \zeta \varphi^{\prime}+K_{1} h^{\prime}+M \delta_{1}\left(\frac{m \delta_{1} f}{1+m^{2} \delta_{1}{ }^{2}}-\right.\right. \\
\left.\left.\frac{\varphi}{1+m^{2} \delta_{1}{ }^{2}}\right)\right] \frac{\theta-\theta_{c}}{\theta_{c}}+\frac{\theta^{\prime} \varphi^{\prime}}{\theta-\theta_{c}}
\end{array} \\
& \quad\left(2+K_{1}\right) h^{\prime \prime}=K_{2}\left(4 h+2 f^{\prime}\right)-4 \zeta h^{\prime}
\end{aligned}
$$

The corresponding boundary conditions are

$$
\left.\begin{array}{l}
\text { As } \eta=0: f=0, \varphi=0, \theta^{\prime}=-1, g=1, h=0 \\
\text { As } \eta \rightarrow \infty: f \rightarrow 0, \varphi \rightarrow 0, g \rightarrow 0, \theta \rightarrow 0, h \rightarrow 0
\end{array}\right\} \ldots
$$

The physical quantities of interest in this problem are the skin -friction coefficient $c_{f}$, Nusselt number $N u$ and Sherwood number $S_{h}$ which indicate physically wall shear stress, rate of heat transfer and rate of mass transfer respectively. For micropolar boundary layer flow, the wall shear stress $\tau_{w}$ is given by

$$
\tau_{w}=\left[(\mu+k) \frac{\partial u}{\partial y}+k N\right]_{y=0}=\rho v_{\infty}\left(\frac{\theta_{c}}{\theta_{c}-\theta(0)}+K_{1}\right) f^{\prime}(0)
$$

The skin -friction coefficient $c_{f}$ can be defined as

$$
c_{f}=\frac{2 \tau_{w}}{\rho U_{0}{ }^{2}}=2\left(\frac{\theta_{c}}{\theta_{c}-\theta(0)}+K_{1}\right) R e^{-1 / 2} f^{\prime}(0)
$$

The Nusselt number is given by

$$
N u=\frac{x q}{\lambda_{\infty}\left(T_{w}-T_{\infty}\right)}=\frac{1}{2} R e^{1 / 2}\left[\frac{\theta_{r}}{\theta_{r}-\theta(0)}\right] \frac{1}{\theta(0)}
$$

The mass flux at the wall is given by

$$
\begin{aligned}
& M_{w}=-D\left[\frac{\partial C}{\partial y}\right]_{y=0} \\
& S_{h}=\frac{S_{c} M_{w} x}{v_{\infty}\left(C_{w}-C_{\infty}\right)}=-\frac{1}{2} R^{1 / 2}\left[\frac{\theta_{c}}{\theta_{c}-\theta(0)}\right] g^{\prime}(0)
\end{aligned}
$$

\section{RESULTS AND DISCUSSIONS}

The equations (19) - (23) together with the boundary conditions (24) are solved for various combination of the parameters involved in the equations using an algorithm based on the shooting method and presented results for the dimensionless primary velocity distribution, secondary velocity distribution, micro-rotation distribution, species concentration distribution, temperature distribution with the variation of different parameters.

Initially solution was taken for constant values of $\mathrm{Sc}=2$, $\mathrm{Ec}=0.10, \operatorname{Pr}=0.70, \delta_{1}=0.10, \mathrm{~m}=1, \mathrm{Gr}=0.50, \mathrm{~K} 1=$ $0.50, \mathrm{~K} 2=0.25, v_{0}=0.25, \mathrm{~K}=0.50, \mathrm{Re}=0.50$ with the viscosity parameter $\theta_{c}$ ranging from -15 to -1 at certain value of $\theta_{r}=-10$. Similarly solutions have been found with varying the thermal conductivity parameter $\boldsymbol{\theta}_{\boldsymbol{r}}$ ranging from 15 to -1 at certain value of $\theta_{c}=-10$ keeping the other values remaining same. Solutions have also been found for different values of Magnetic parameter $(M)$, dimensionless reference temperature corresponding to thermal conductivity parameter $\left(\theta_{r}\right)$, dimensionless reference temperature corresponding to viscosity parameter $\left(\theta_{c}\right)$, Prandtl number $\left(P_{r}\right)$, Eckert Number $(\mathrm{Ec})$, the coupling constant parameter $\left(K_{1}\right)$ Suction parameter $\left(v_{0}\right)$, permeability parameter $(\mathrm{K})$ and Schmidt number $(\mathrm{Sc})$.

The variations in primary velocity distribution, secondary velocity distribution, temperature distribution, species concentration distribution and micro-rotation distribution are illustrated in figures (1) - (15) with the variation of different parameters.

The figures (1) - (9) represent the variations in dimensionless primary velocity distribution and secondary velocity distribution with Magnetic parameter, dimensionless reference temperature corresponding to viscosity parameter and thermal conductivity parameter due to heating $(\mathrm{Gr}<0)$ and cooling of the plate $(\mathrm{Gr}>0)$, coupling constant parameter, permeability parameter and suction parameter. Figures (1) and (2) represent the variations of primary velocity and secondary velocity distribution with the variation of Magnetic parameter. We observe that primary velocity decreases with the increasing values of $\mathrm{M}$. It is because that the application of transverse magnetic field will result a resistive force (Lorentz force) similar to drag force, which tends to resist the fluid flow and thus reducing its velocity. We have seen that secondary velocity increases for $\eta<1$ and then decreases for increasing value of $M$. Figures (3) - (6) represent the variations of primary velocity with the variation of $\theta_{c}$ and $\theta_{r}$ due to heating $(\mathrm{Gr}<0)$ and cooling of the plate $(\mathrm{Gr}>0)$ respectively. We observe that both primary and secondary velocity decreases with the increasing values of $m$. From figure (7) we observe that primary velocity decreases with increasing values of $v_{0}$. From figure (8) it is seen that primary velocity decreases when $\eta<1.5$ and then increases with the increasing value of coupling parameter $K_{1}$. From figure (9) we have observed that primary velocity increases with increasing values of permeability parameter. The figures (10) and (11) represent the variations in dimensionless temperature profile $\boldsymbol{\theta}(\eta)$ with the variation of Prandtl number and Eckert number respectively. From figure (10) we have observed that temperature decreases with the increasing values of $P_{r}$.It is due to the reason that with the increasing values of the Prandtl number the viscosity increases and as a result temperature decreases. From figure (11)) we have seen that temperature increases when both the values of $E c$. Figure (12) displays the distribution representing concentration 
profile within the boundary layer with the variation of $S c$, It is observed that concentration decreases with the increasing values of $S c$ The profiles have the common feature that the concentration decreases in a monotone fashion from the surface to a zero value far away in the free stream. As $S c$ is the connecting link between velocity and concentration

\subsection{Figures}

profiles, therefore with the increasing value of $S c$ molecular mass diffusivity decreases and as a result concentration decreases. Figures (13) - (15) display the distribution representing micro-rotation within the boundary layer with the variation of $K_{1}, \theta_{c}$ and $\theta_{r}$.

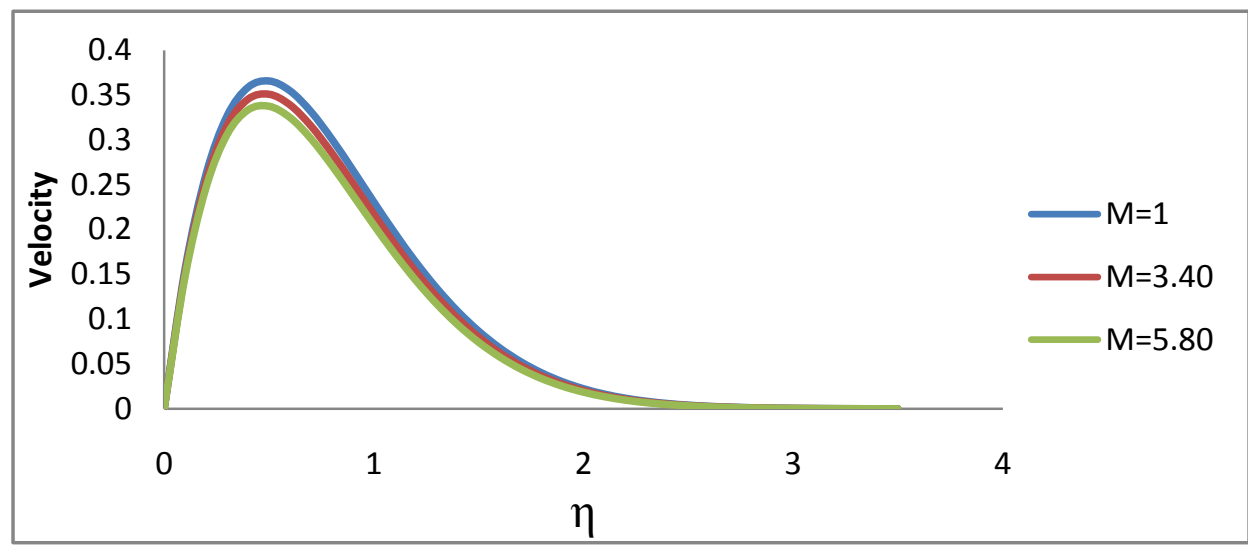

Figure 1: Variation of primary velocity distribution with $M$

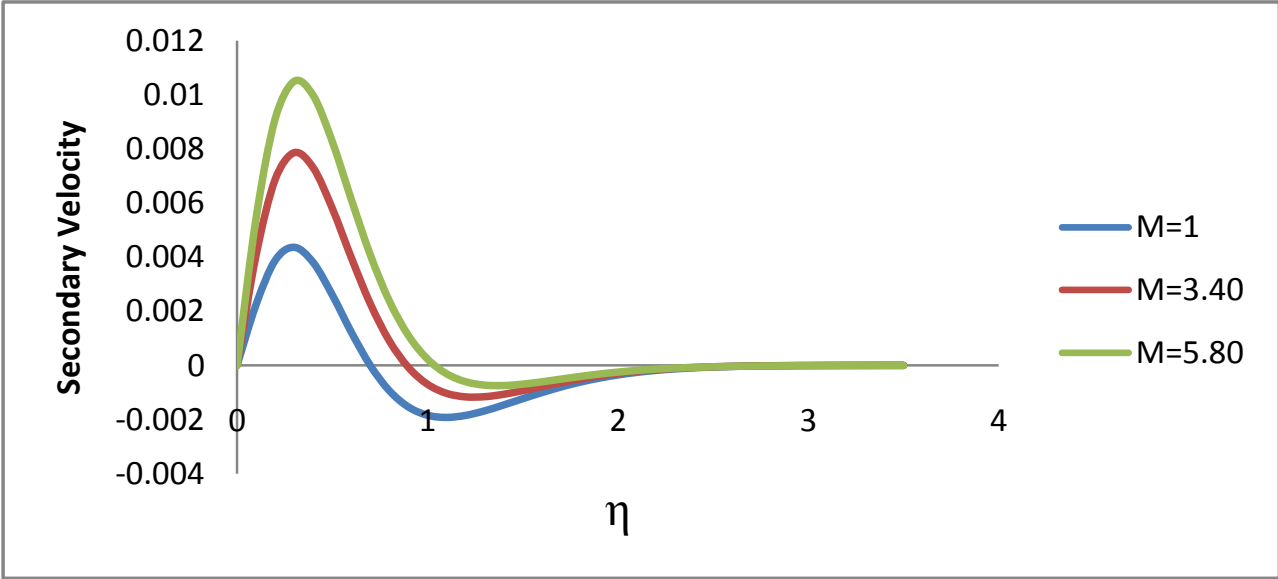

Figure 2: Variation of secondary velocity distribution with $M$

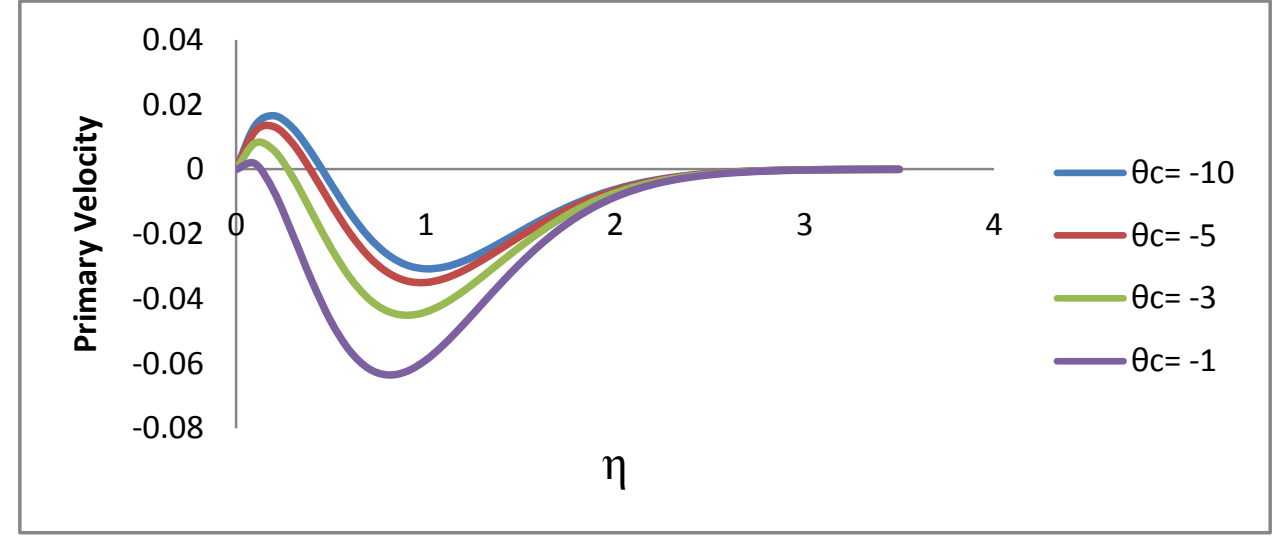

Figure 3: Variation of primary velocity distribution with $\theta_{c}$ due to heating of the plate $(\mathrm{Gr}<0)$ 


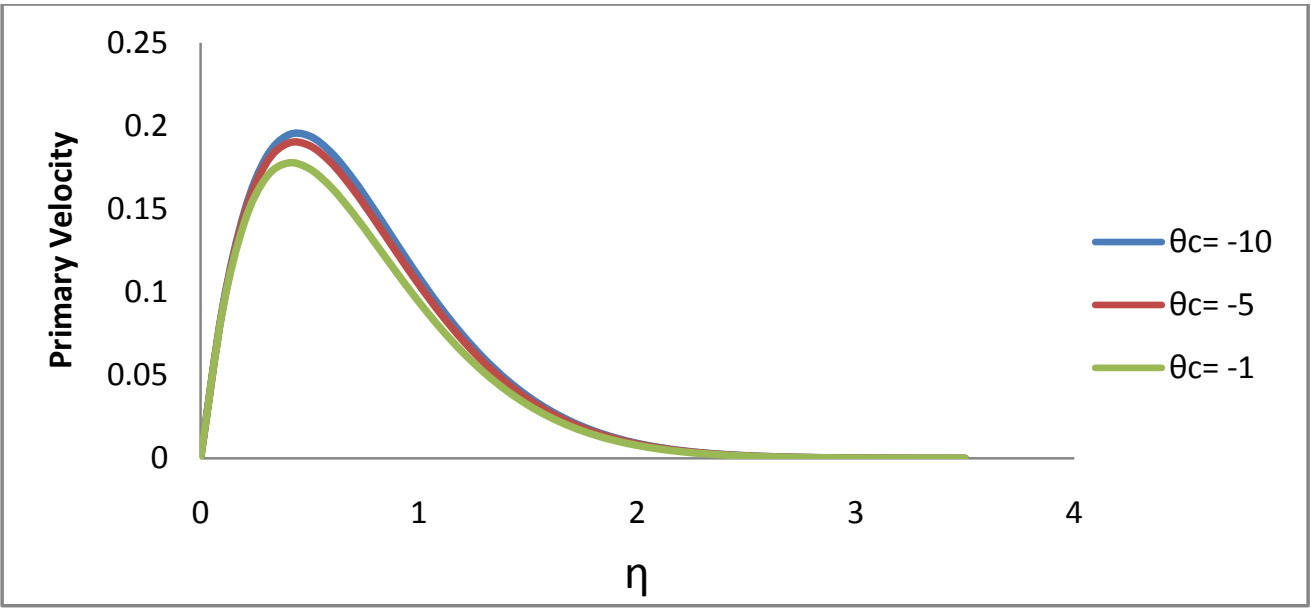

Figure 4: Variation of primary velocity distribution with $\boldsymbol{\theta}_{c}$ due to cooling of the plate (Gr>0)

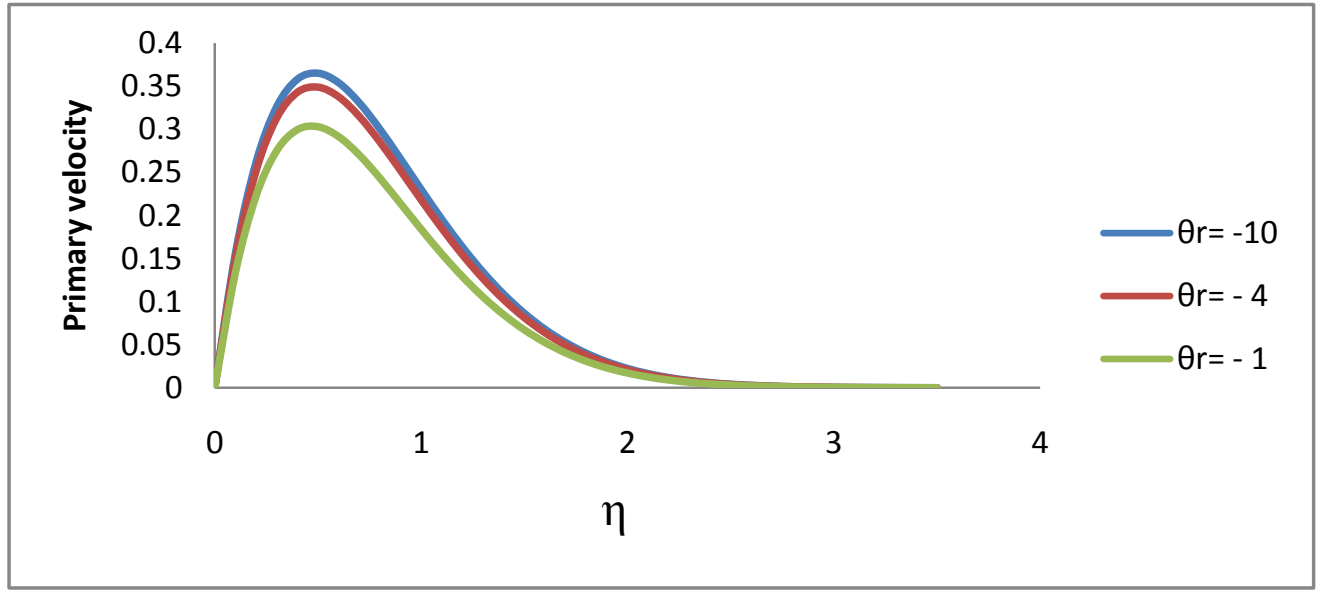

Figure 5: Variation of primary velocity distribution with $\theta_{r}$ due to cooling of the plate (Gr>0)

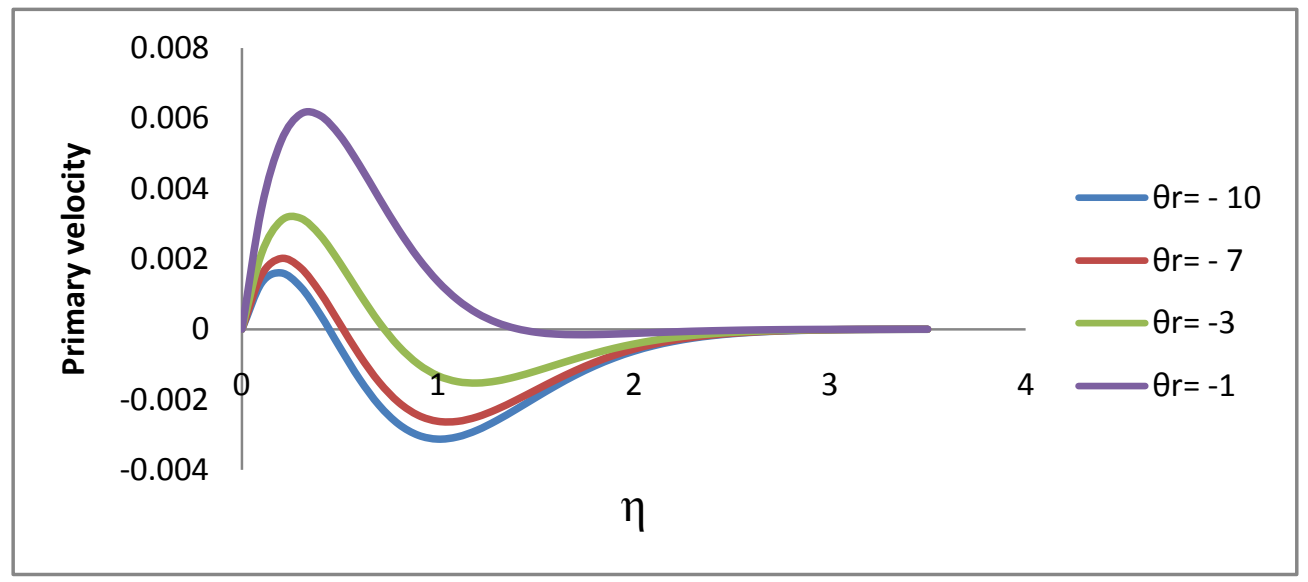

Figure 6: Variation of primary velocity distribution with $\theta_{r}$ due to heating of the plate $(\mathrm{Gr}<0)$ 


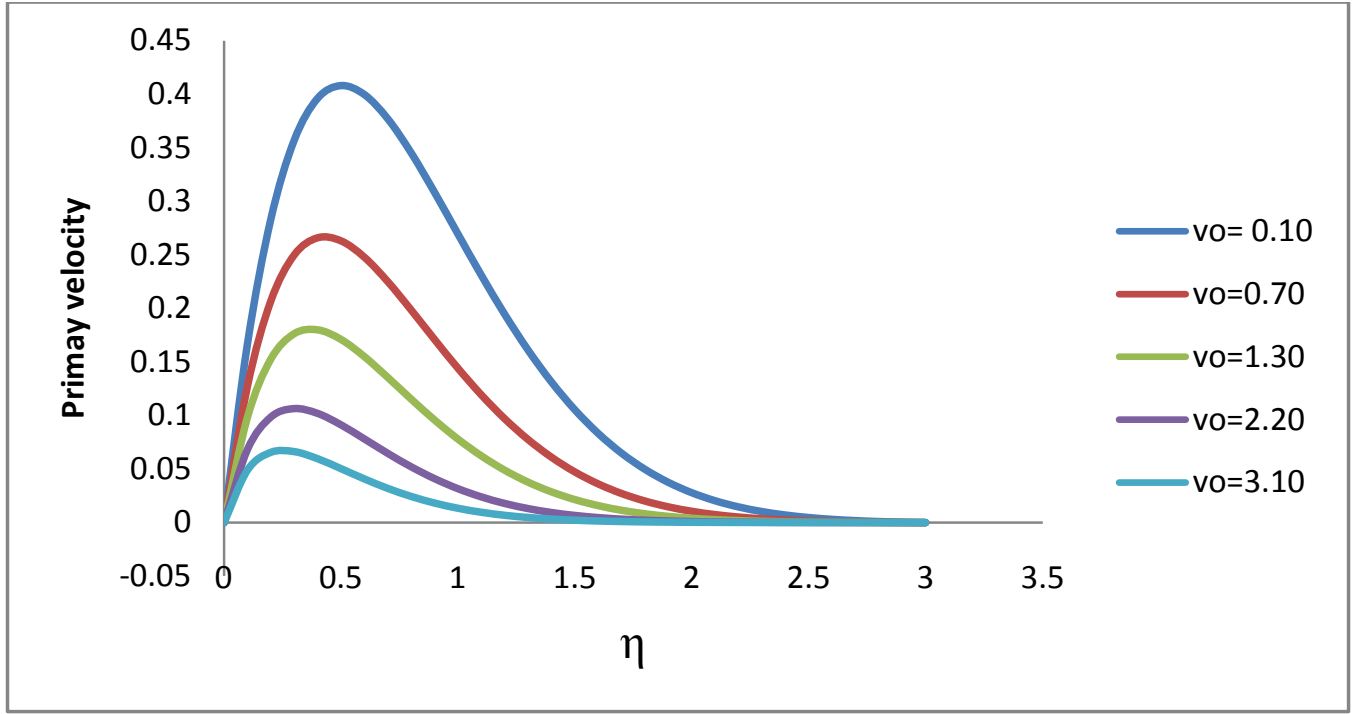

Figure 7: Variation of primary velocity distribution with suction parameter $\boldsymbol{v}_{0}$

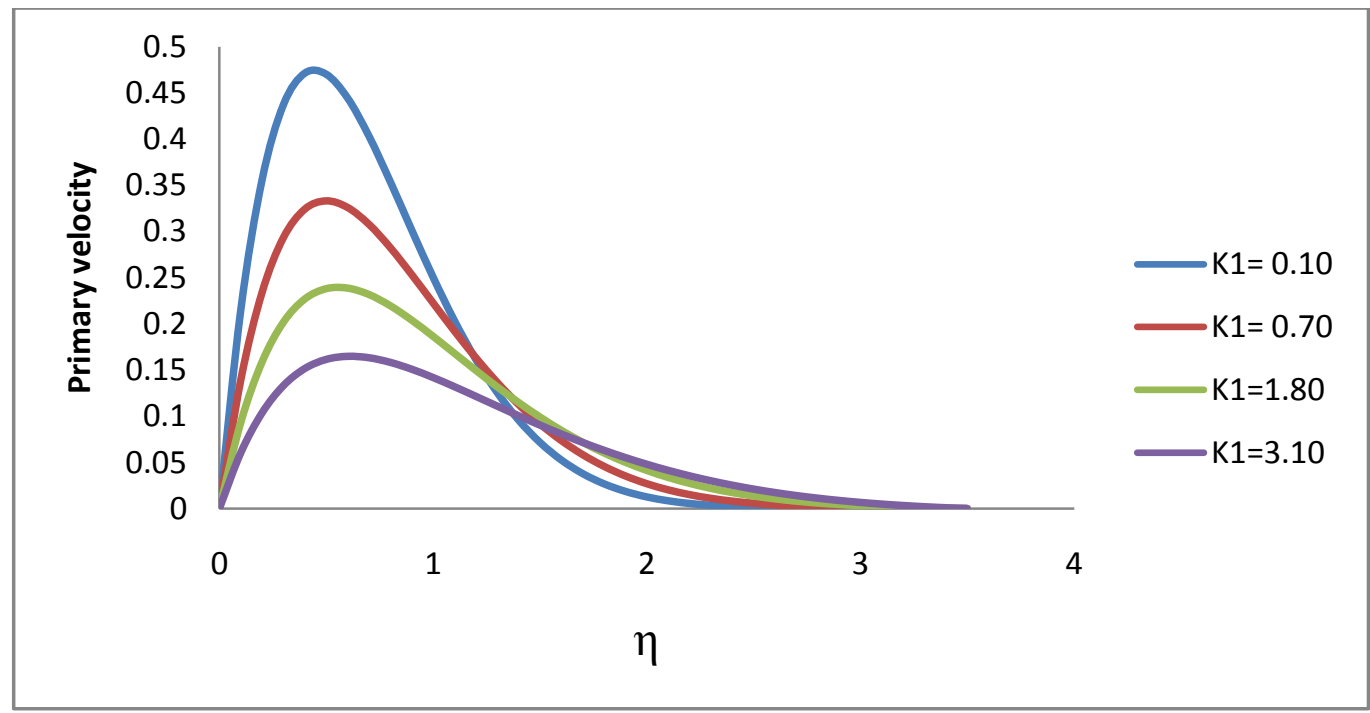

Figure 8: Variation of primary velocity distribution with K1

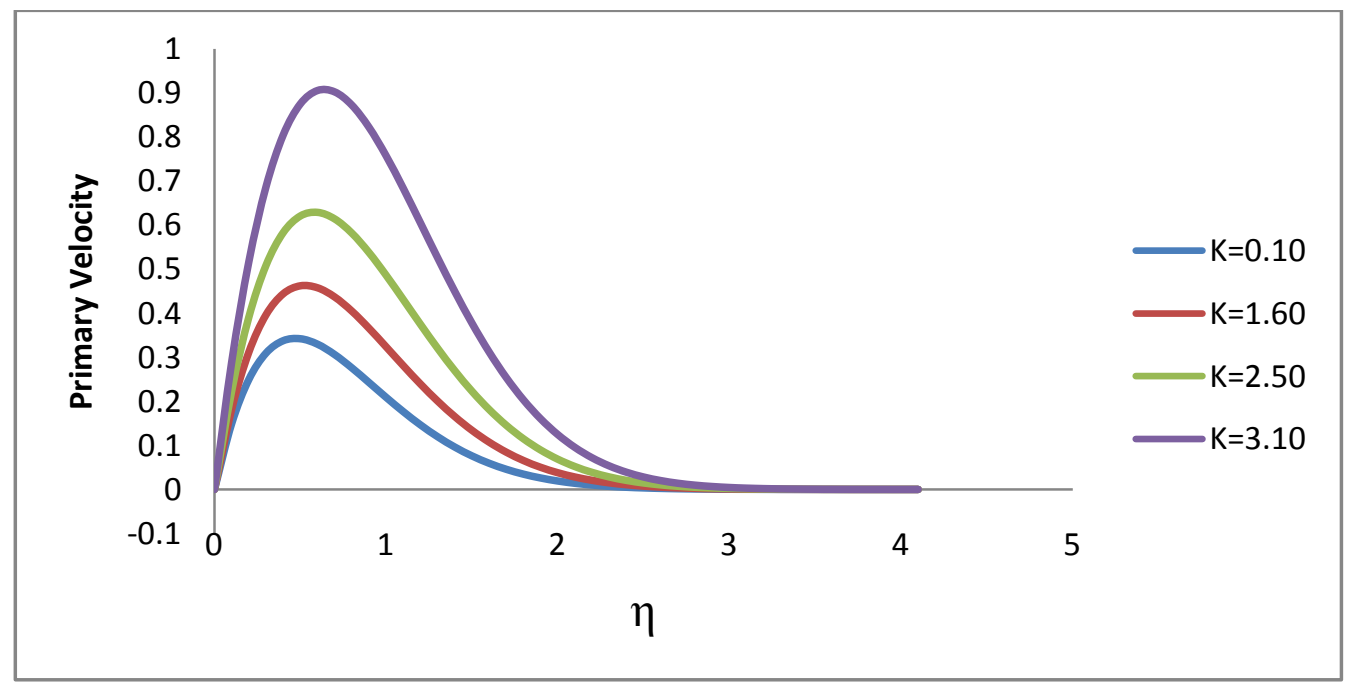

Figure 9: Variation of primary velocity distribution with $\mathrm{K}$ 


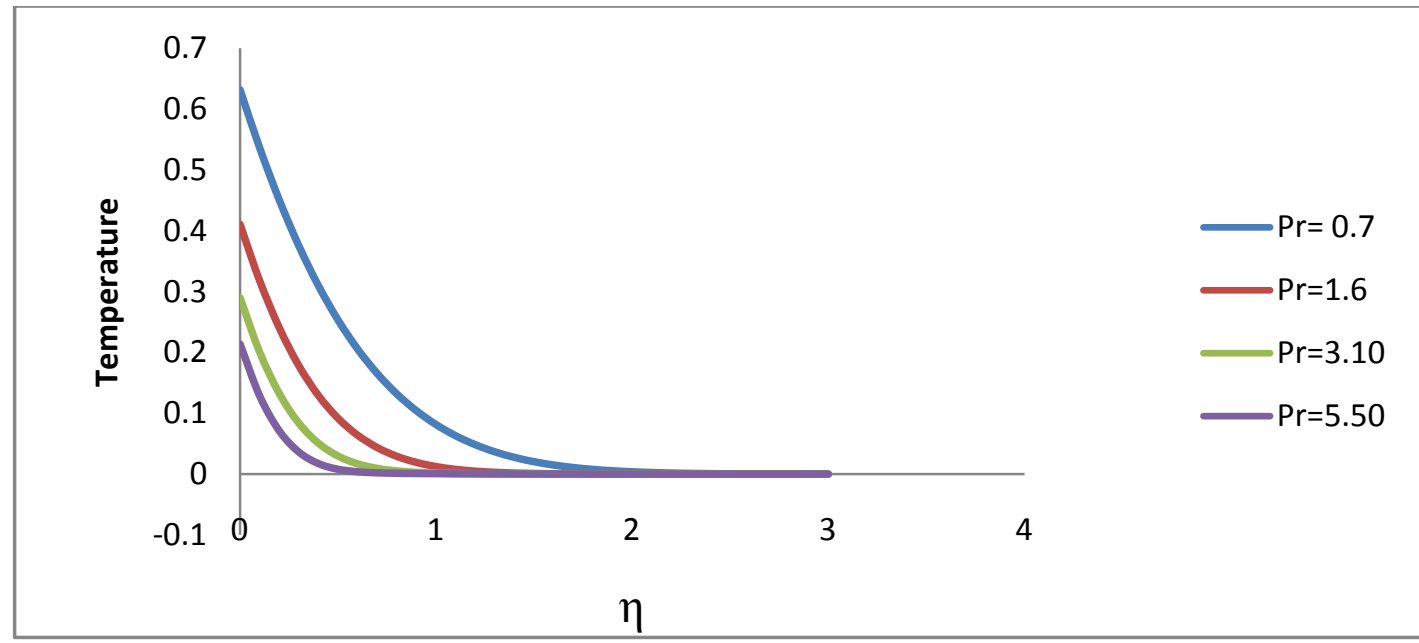

Figure 10: Variation of temperature distribution with $\mathrm{Pr}$

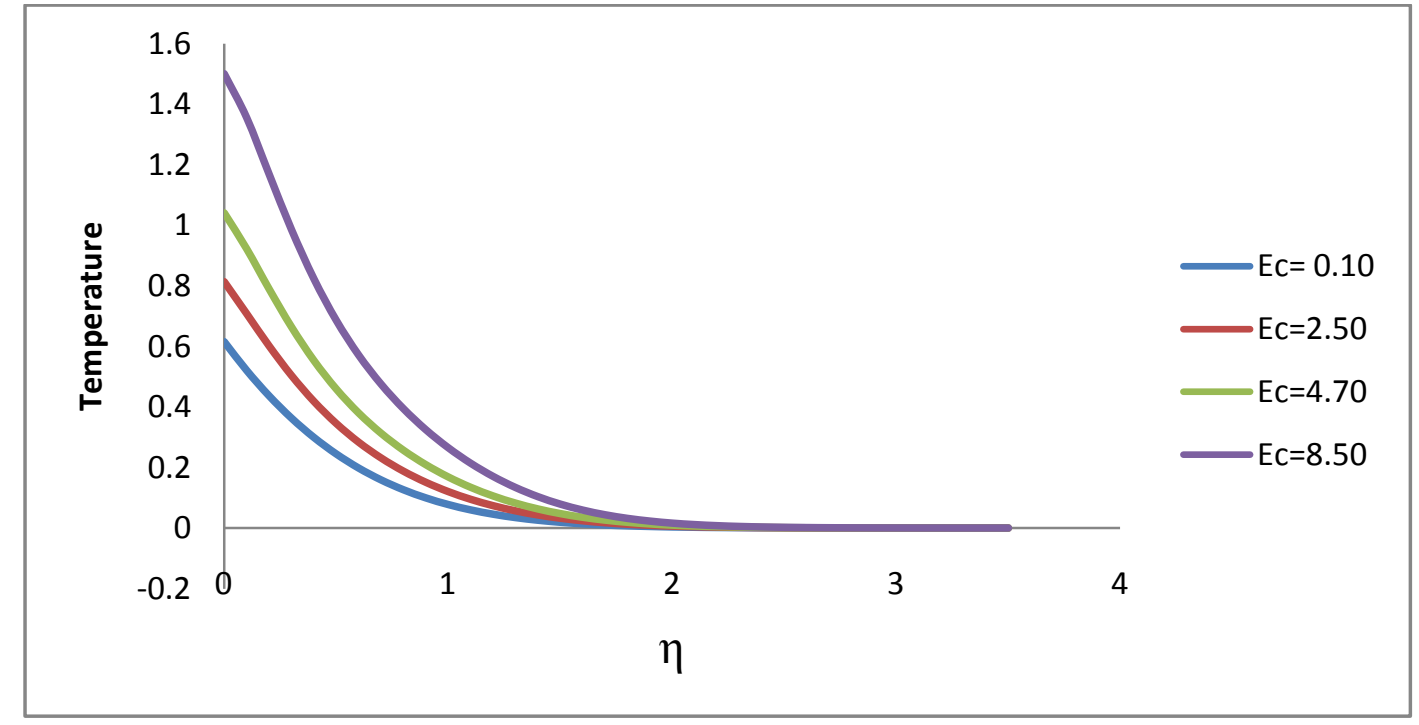

Figure 11: Variation of temperature distribution with Ec

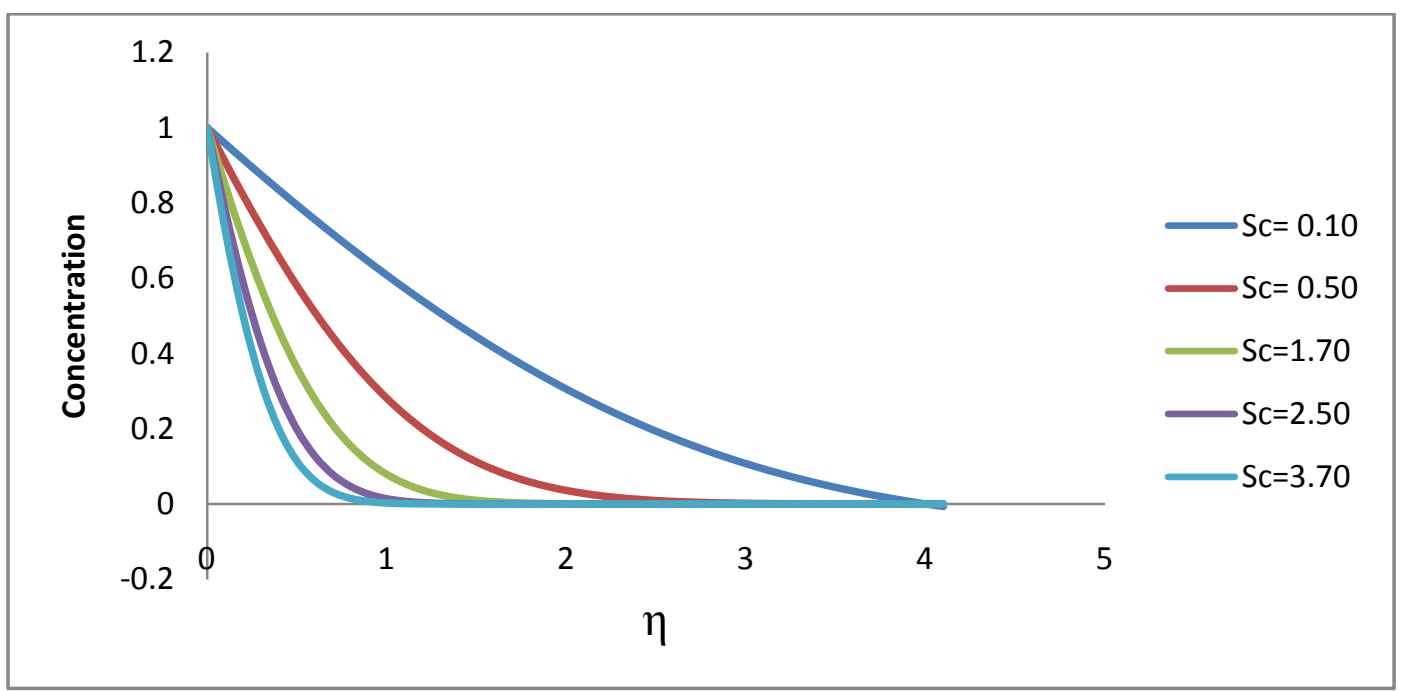

Figure 12: Variation of concentration distribution with Sc 


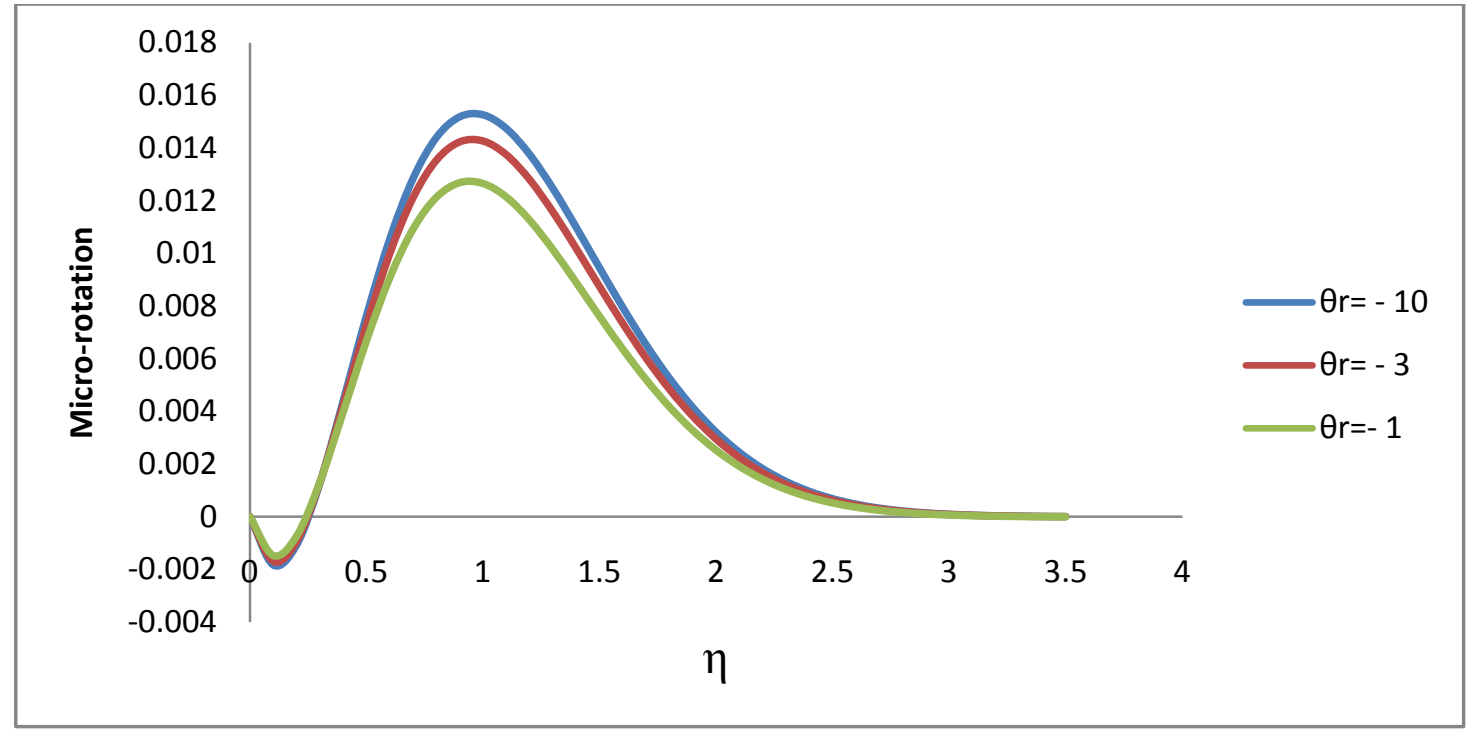

Figure 13: Variation of micro-rotation distribution with $\theta_{r}$

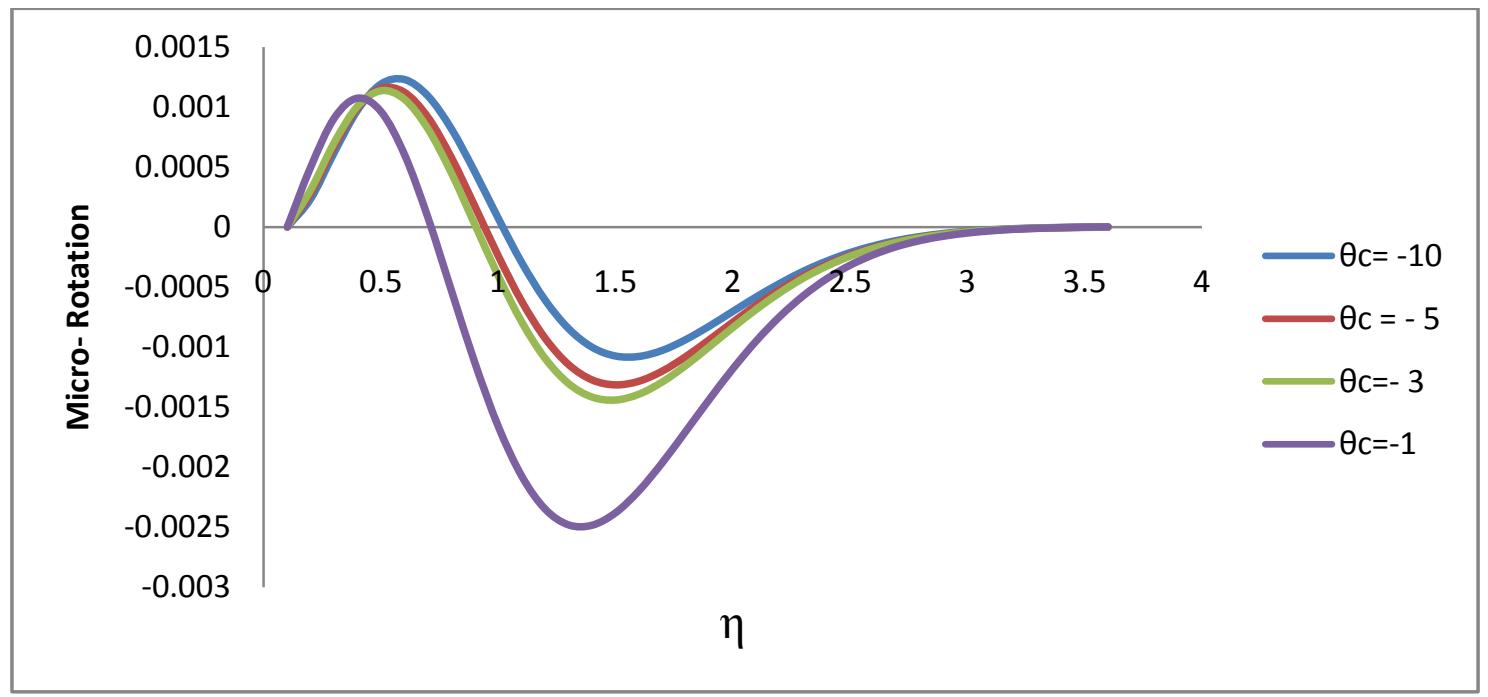

Figure 14: Variation of micro-rotation distribution with $\boldsymbol{\theta}_{c}$

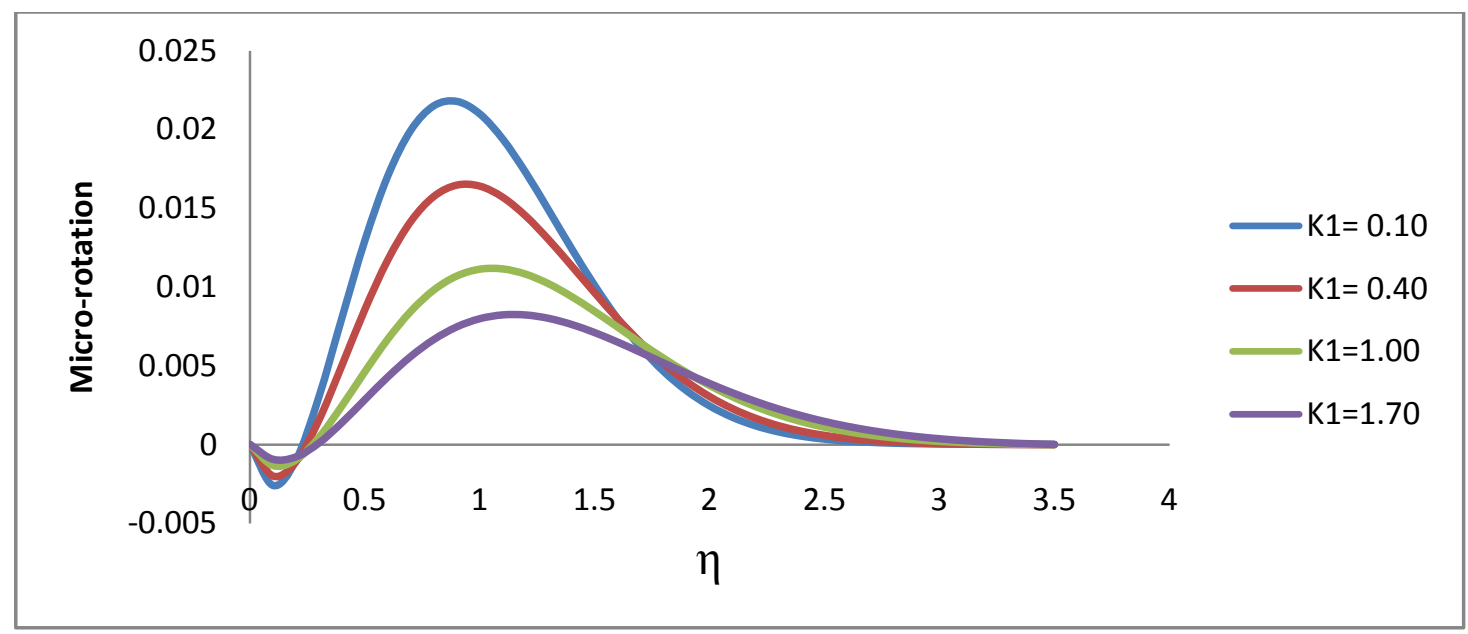

Figure 15: Variation of Micro-rotation distribution with $K_{1}$ 


\subsection{Tables}

Finally the effects of the above mentioned parameters on the values of $f^{\prime}(0), \mathrm{g}^{\prime}(0), \varphi^{\prime}(0), \mathrm{h}^{\prime}(0), \theta(0), C_{f}, N u$ and $\boldsymbol{S}_{\boldsymbol{h}}$ are shown in the tables (1) - (3). We consider $\mathrm{Sc}=2, \theta_{c}=-10, \mathrm{Ec}=0.10, \theta_{r}=-10, \mathrm{Pr}=0.70, \delta_{1}=0.10, \mathrm{~m}=1.00, G c=0.05, \mathrm{Gr}=0.50, \mathrm{~K}_{1}=0.50, \mathrm{~K}_{2}=0.25$, $\mathrm{v} 0=0.25, \mathrm{~K}=0.50, \mathrm{Re}=0.50$. The behavior of these parameters is self evident from the tables and hence any further discussions about them seem to be redundant.

Table 1: Numerical values with the variation of $M$ and $G c$

\begin{tabular}{|c|c|c|c|c|c|c|c|c|c|}
\hline$M$ & $G c$ & $f^{\prime}(0)$ & $\varphi(0)$ & $\theta(0)$ & $g(0)$ & $h(0)$ & $c_{f}$ & $N u$ & $S_{h}$ \\
\hline \multirow{4}{*}{1} & 0.1 & 0.099866 & 0.001317 & 0.607654 & -2.0101 & -0.00209 & 0.423756 & -227.226 & 0.234495 \\
\hline & 1 & 0.279443 & 0.003346 & 0.608122 & -2.01016 & -0.00509 & 1.185979 & -93.0704 & 0.234477 \\
\hline & 2.5 & 0.578903 & 0.006732 & 0.609849 & -2.01038 & -0.0101 & 2.457733 & -46.8875 & 0.234414 \\
\hline & 4 & 0.878577 & 0.010121 & 0.612761 & -2.01075 & -0.01512 & 3.731248 & -31.3227 & 0.234306 \\
\hline \multirow[t]{4}{*}{2.5} & 0.1 & 0.098366 & 0.002128 & 0.607651 & -2.0101 & -0.00203 & 0.417389 & -233.551 & 0.234495 \\
\hline & 1 & 0.275861 & 0.005324 & 0.608103 & -2.01016 & -0.00496 & 1.170766 & -95.4922 & 0.234478 \\
\hline & 2.5 & 0.571842 & 0.010654 & 0.609774 & -2.01037 & -0.00985 & 2.427717 & -48.0792 & 0.234416 \\
\hline & 4 & 0.868027 & 0.015991 & 0.612593 & -2.01073 & -0.01475 & 3.686355 & -32.113 & 0.234312 \\
\hline \multirow{4}{*}{4.5} & 0.1 & 0.096494 & 0.003 & 0.607647 & -2.0101 & -0.00196 & 0.409444 & -241.875 & 0.234495 \\
\hline & 1 & 0.271375 & 0.007473 & 0.60808 & -2.01015 & -0.0048 & 1.151717 & -98.6718 & 0.234479 \\
\hline & 2.5 & 0.562991 & 0.014935 & 0.609683 & -2.01036 & -0.00954 & 2.390091 & -49.6427 & 0.23442 \\
\hline & 4 & 0.854798 & 0.022405 & 0.612388 & -2.0107 & -0.01429 & 3.630061 & -33.1496 & 0.23432 \\
\hline
\end{tabular}

Table 2: Numerical values with the variation of $M$ and $P_{r}$

\begin{tabular}{|c|c|c|c|c|c|c|c|c|c|}
\hline $\mathrm{M}$ & $\operatorname{Pr}$ & $\mathrm{f}^{\prime}(0)$ & $\varphi(0)$ & $\theta(0)$ & $\mathrm{g}(0)$ & $\mathrm{h}(0)$ & $c_{f}$ & $N u$ & $S_{h}$ \\
\hline 2 & 0.7 & 0.17757 & 0.003113 & 0.605664 & -2.00986 & -0.00335 & 0.753533 & -140.664 & 0.234567 \\
\hline & 2.7 & 0.119329 & 0.001834 & 0.295559 & -1.97216 & -0.00193 & 0.506336 & -252.139 & 0.246251 \\
\hline \multirow{2}{*}{5.7} & 0.107829 & 0.00165 & 0.193626 & -1.95841 & -0.00175 & 0.457532 & -280.941 & 0.25046 \\
\hline & 7.2 & 0.102641 & 0.001493 & 0.167307 & -1.95475 & -0.00152 & 0.435514 & -323.121 & 0.251578 \\
\hline \multirow{2}{*}{4} & 0.7 & 0.174502 & 0.004635 & 0.605654 & -2.00986 & -0.00324 & 0.74051 & -145.5 & 0.234567 \\
\cline { 2 - 9 } & 2.7 & 0.117626 & 0.002703 & 0.295554 & -1.97216 & -0.00187 & 0.499108 & -260.071 & 0.246251 \\
\hline & 5.7 & 0.106284 & 0.00243 & 0.19362 & -1.95841 & -0.00169 & 0.450976 & -289.808 & 0.25046 \\
\hline & 7.2 & 0.101448 & 0.002184 & 0.167361 & -1.95476 & -0.00149 & 0.43045 & -330.438 & 0.251576 \\
\hline
\end{tabular}


Table 3: Numerical values with the variation of $M$ and $\boldsymbol{\theta}_{\boldsymbol{r}}$

\begin{tabular}{|c|c|c|c|c|c|c|c|c|c|}
\hline $\mathrm{M}$ & $\theta_{r}$ & $\mathrm{f}^{\prime}(0)$ & $\varphi(0)$ & $\theta(0)$ & $\mathrm{g}(0)$ & $\mathrm{h}(0)$ & $c_{f}$ & $N u$ & $S_{h}$ \\
\hline \multirow{2}{*}{1} & -10 & 0.17926 & 0.002212 & 0.60568 & -2.00986 & -0.00341 & 0.760707 & -138.224 & 0.234566 \\
\cline { 2 - 9 } & -5 & 0.175161 & 0.00215 & 0.584224 & -2.00726 & -0.00331 & 0.74331 & -135.266 & 0.235347 \\
\cline { 2 - 9 } & -1 & 0.156217 & 0.001867 & 0.480793 & -1.99455 & -0.00285 & 0.6629 & -118.387 & 0.239183 \\
\hline \multirow{2}{*}{4} & -10 & 0.174555 & 0.004646 & 0.605664 & -2.00986 & -0.00324 & 0.740732 & -145.538 & 0.234567 \\
\cline { 2 - 9 } & -5 & 0.170603 & 0.00451 & 0.584209 & -2.00726 & -0.00314 & 0.72396 & -142.395 & 0.235347 \\
\hline & -1 & 0.152322 & 0.003892 & 0.480783 & -1.99455 & -0.00271 & 0.646363 & -124.506 & 0.239183 \\
\hline
\end{tabular}

\section{CONCLUSION:}

In this study the effects of temperature dependent viscosity and thermal conductivity on magneto hydrodynamic unsteady free convective heat and mass transfer flow of an incompressible micropolar fluid through a porous medium along an infinite vertical plate is studied. The results demonstrate clearly that the viscosity and thermal conductivity parameters along with the other parameters viz. mass transfer parameter, coupling constant parameter, Prandtl number, Schmidt number, Hall parameter and magnetic parameter have significant effects on velocity, secondary velocity, temperature, concentration and micro-rotation distributions within the boundary layer. Thus assumption on constant properties may cause a significant error in flow problem.

\section{NOMENCLATURES}

$\beta=$ Volumetric coefficient of thermal expansion

$\beta^{*}=$ Volumetric co-efficient of expansion with concentration

$g_{0}=$ Gravitational acceleration

$\lambda=$ Thermal conductivity

$\mu=$ Dynamic viscosity

$\mu_{\infty}=$ Dynamic viscosity of the ambient fluid

$v=$ Kinematic viscosity

$v_{\infty}=$ Kinematic Viscosity of the ambient fluid

$\kappa=$ Vortex viscosity

$\gamma=$ Spin-gradient or micro rotation viscosity

$c_{\mathrm{p}}=$ Specific heat

$\mathrm{v}_{0}=$ Suction parameter

$\eta=$ Dimensionless co-ordinates

$u=$ Velocity in the $x$-direction

$v=$ Velocity in the $y$-direction

$w=$ Secondary velocity

$U_{0}=$ Reference velocity

$f=$ Dimensionless velocity

$\varphi=$ Dimensionless Secondary velocity

$h=$ Dimensionless micro-rotation $g=$ Dimensionless species concentration

$\theta=$ Dimensionless temperature

$\theta_{c}=$ Dimensionless reference temperature corresponding to viscosity parameter

$\theta_{r}=$ Dimensionless reference temperature corresponding to thermal conductivity parameter

$T=$ Temperature

$C=$ Species concentration

$T_{\infty}=$ Ambient temperature

$T_{w}=$ Wall temperature

$C_{w}=$ Species concentration at the wall

$C_{\infty}=$ Species concentration far from the wall

$j=$ Micro-inertia density

$\sigma=$ Electrical conductivity

$\rho=$ Density

$D=$ Thermal molecular diffusivity

$G_{r}=$ Local Grashoff number for heat transfer

$G_{c}=$ Local Grashoff number for mass transfer

$S_{c}=$ Schmidt number

$P_{r}=$ Prandtl number

$R_{e}=$ Local Reynolds number

$M=$ Hartmann Number

$m=$ Hall parameter

$B_{0}=$ Constant magnetic field intensity

$K=$ Permeability parameter

$K_{1}=$ Coupling constant parameter

$q_{w}=$ Heat transfer from the plate

$C_{\mathrm{f}}=$ Skin-friction coefficient

$\mathrm{Nu}=$ Nusselt number

$S_{h}=$ Sherwood number

Subscripts: 
$\mathbf{w}$, the condition at the wall

$\infty$, the condition far away from the surface

\section{Superscripts:}

, Differentiation with respect to $\eta$

\section{REFERENCES}

[1] Eringen A.C., Theory of micropolar fluids, J. Math. Mech., Vol.16, (1966), pp.1-18.

[2] Gorla R.S.R., Takhar H.S., Slaouti A., Magneto hydrodynamic free convection boundary layer flow of a thermo micropolar fluid over a vertical plate, Int. J. Eng. Sci., Vol. 36(1998) pp. 315-327.

[3] Lai F.C. and Kulacki F.A., The effect of variable viscosity on convective heat and mass transfer along a vertical surface in saturated porous medium, Int. J. Heat and Mass Transfer, Vol. 33(1991) pp.1028-1031.

[4] Katagiri M., The Effect of Hall Currents on the Magneto hydrodynamic Boundary Layer Flow past a Semi-infinite Flat Plate, Journal of the Physical Society of Japan, Vol. 27, No 4(1969), pp. 1051-1059.

[5] Hossain M A, Effect of Hall Current on Unsteady Hydro magnetic Free-convection Flow near an Infinite Vertical Porous Plate, Journal of the Physical Society of Japan, Vol. 55, No. 7 (1986), pp. 2183-2190.
[6] Sattar M.A. and Hossain M. M., Unsteady Hydromegnetic free convection flow with hall current and mass Transfer along an accelerated porous Plate with Time Dependent Temperature and Concentration, Can. J. Phy., Vol.70,pp 369-374, 1992.

[7] Sattar M.A. Rahman, M. M and Alam, M. M., Free convection flow heat Transfer through a porous vertical Plate immersed in a porous medium with variable suction, J. Energy Heat And Mass Transfer, Vol.22, pp 17-21, 2000.

[8] Sarma U., Hazarika G. C. , Effects of variable viscosity and thermal conductivity on combined free-forced convection and mass transfer flow passed a vertical porous plate, Antarctica J. Math., Vol.7, No. 4 (2010), pp.461-472.

[9] Sarma U., Hazarika G. C., Effects of variable viscosity and thermal conductivity on free convective heat and mass transfer flow with constant heat flux through a porous medium, J. Comp. \& Math. Sci., Vol.1, No. 2 (2010), pp.163-170.

[10] Schlichting H., Boundary layer Theory, $6^{\text {th }}$ Edn., McGraw-Hill, New York, 1968.

[11] Shercliff J. A., A textbook of magneto hydrodynamics, pergamon press inc., New York, 1965. 